\section{Impact on participation and autonomy: test of validity and reliability for older persons}

\author{
Isabelle Ottenvall Hammar,1-4 \\ Christina Ekelund, 1,3 Katarina \\ Wilhelmson, 3-5 Kajsa Eklund1,3,4 \\ 1Department of Clinical Neuroscience and \\ Rehabilitation, Institute of Neuroscience \\ and Physiology, The Sahlgrenska \\ Academy, University of Gothenburg; \\ 2Department of Physiotherapy and \\ Occupational therapy, The Sahlgrenska \\ University Hospital; 3Vårdalinstitutet, \\ The Swedish Institute for Health \\ Sciences, Universities of Gothenburg and \\ Lund; 4Centre of Aging and Health-AGE- \\ CAP, University of Gothenburg; \\ 5 Department of Geriatrics, The \\ Sahlgrenska University Hospital, Sweden
}

\section{Abstract}

In research and healthcare it is important to measure older persons' self-determination in order to improve their possibilities to decide for themselves in daily life. The questionnaire Impact on Participation and Autonomy (IPA) assesses self-determination, but is not constructed for older persons. The aim of this study was to examine the validity and reliability of the IPA-S questionnaire for persons aged 70 years and older. The study was performed in two steps; first a validity test of the Swedish version of the questionnaire, IPA-S, followed by a reliability test-retest of an adjusted version. The validity was tested with focus groups and individual interviews on persons aged 77 88 years, and the reliability on persons aged 70-99 years. The validity test result showed that IPA-S is valid for older persons but it was too extensive and the phrasing of the items needed adjustments. The reliability test-retest on the adjusted questionnaire, IPA- Older persons (IPA-0), showed that 15 of 22 items had high agreement. IPA- 0 can be used to measure older persons' self-determination in their care and rehabilitation.

\section{Introduction}

According to both the United Nations and the European Union declarations, healthcare targeting older persons should be built on respect for the individual's self-determination.1,2 Older persons' with multiple diseases and/or affected ability to perform activi- ties of daily living (ADL), often meet an abundance of health care professionals. ${ }^{3}$ To exercise self-determination and to be in control in life are important for health and well-being in old age. ${ }^{4}$ It is important that older persons are allowed to make and influence decisions over their own daily life for their health and wellbeing, 4,5 especially for those persons who are dependent on others. ${ }^{4}$ Older persons right to self-determination is known by professionals. 4 In spite of this, recent studies indicate that older persons' self-determination is not fully respected within the healthcare sector. ${ }^{6-9}$

Self-determination can be described as the ability to think, choose, decide and act independently. 10 The concepts of autonomy and self-determination are often used synonymously. ${ }^{11}$ When the concept is defined in a context of persons with affected ability to perform ADL independently, the concept according to Collopy ought to be divided into decisional autonomy and executional autonomy. 12 Decisional autonomy is the capacity to make personal choices, irrespectively of the person's ability to perform their own choices, while executional autonomy is the performance of the choices. ${ }^{12}$ Due to Cardol et al. ${ }^{13}$ self-determination is equated to decisional autonomy. Henceforth, in this article the concept of selfdetermination will be used as a synonymous to decisional autonomy.

One questionnaire that assesses self-determination is the generic self-report questionnaire Impact on Participation and Autonomy (IPA), but it is not constructed for the older population. The first version of the IPA was developed in the Netherlands and has been psychometrically tested in terms of homogeneity and construct validity for persons with various disabling conditions with the mean age about 48 years (range 23-79 years). 14 The original IPA items have shown good construct, divergent and convergent validity, and have also shown good ability to sense changes over time, homogeneity and test-retest reliability. ${ }^{14-}$ 16 The Swedish version IPA-S, equal to the original version of IPA, 17 has been tested for validity and reliability for persons mean age 52 years (range 17-84 years) with spinal cord injury, showing promising psychometric results. ${ }^{17}$ Since the original IPA based its psychometric properties on persons $<79$ and the IPA-S on a single chronic condition, further psychometric studies for older persons are needed. To our knowledge, there is no other instrument to assess older persons' self-determination.

The aim of this study was to examine the validity and reliability of the IPA-S questionnaire for persons aged 70 years and older.
Correspondence: Isabelle Ottenvall Hammar, Department of Clinical Neuroscience and Rehabilitation, The Sahlgrenska Academy at University of Gothenburg, Arvid Wallgrens backe, House 2, Box 455, 40530 Gothenburg, Sweden. Tel.: +46.031 .786 .5788$

E-mail: isabelle.o-h@neuro.gu.se

Key words: impact on participation and autonomy, occupational therapy, rehabilitation, selfdetermination, test-retest.

Acknowledgments: the authors would like to thank Mieke Cardol for valuable feed-back on the manuscript and for examining that IPA-0 fulfils the intent of IPA.

Contributions: the authors contributed equally.

Conflict of interests: the authors declare no potential conflict of interests.

Funding: this study was supported by grants from the Research and Development Council of the County of Gothenburg and South of Bohuslän and Vårdalinstitutet, the Swedish Institute for Health Sciences.

Conference presentation: this study has been presented at the $21^{\text {st }}$ Nordic Congress of Gerontology in Copenhagen, Denmark in June 10th $-13^{\text {th }}, 2012$ and at a National conference in occupational therapy (AT-Forum) in Stockholm, Sweden in May 24th-25th 2013.

Received for publication: 12 July 2014. Revision received: 14 September 2014 Accepted for publication: 14 September 2014.

This work is licensed under a Creative Commons Attribution NonCommercial 3.0 License (CC BYNC 3.0).

(C) Copyright I.O. Hammar et al., 2014

Licensee PAGEPress, Italy

Health Psychology Research 2014; 2:1825

doi:10.4081/hpr.2014.1825

\section{Materials and Methods}

\section{Research design}

The present study was performed in two steps; a validity test and a reliability testretest, (Figure 1). First the face- and content validity of the IPA-S was tested using focus group discussions and individual interviews. An expert panel $(n=4)$ analyzed and discussed the results, 18 which resulted in an adjustment of the IPA-S called IPA-0 (Older persons). The expert panel had knowledge in geriatrics $(n=1)$, occupational therapy $(n=3)$, instrument development $(\mathrm{n}=2)$, in interviewing older persons $(n=4)$ and knowledge in focus groups methodology $(n=3)$. The expert panel 
were selected persons from the research group covering a broad relevant expertise area of frail older persons. The reliability of the IPA-0 was tested with a test-retest with Svensson's method. 19,20

\section{Questionnaire}

The IPA-S was used in the validity test. The original IPA addresses the following five dimensions: autonomy indoors (seven items), family role (seven items), autonomy outdoors (five items), social relationship (six items) and work and education (six items), in total 31 items. The questionnaire has five answer alternatives: very good, good, fair, poor or very poor and the respondents score the items by themselves. At the end of each dimension a question regarding how perceived health problem affects perceived self-determination is answered with: no problems, minor problems or severe problems. Higher scores represent severe problems. ${ }^{15}$

\section{Participants}

Inclusion criteria were community living persons aged 70 years and older, dependent on another person in at least one ADL-activity according to the ADL Staircase. ${ }^{21}$ Before asked to participate in the study, they were assessed by healthcare professionals to be cognitively intact. The healthcare professionals also invited them to participate in the study. In the validity test, eligible persons were recruited from a community based service centre, a convenient way to find dependent, frail older persons living at home. In total, 13 of the 15 persons who were asked to participate actually participated. Nine persons accepted to participate in focus group discussions forming two groups $(4+5)$, four persons accepted to participate in individual interviews. All lived in ordinary housing and home care service varied from having some assistance with heavy shopping by siblings, to four times daily formal home care service. All had at least one chronic condition such as heart failure, arthritis, vertigo or had residues after stroke or cancer (Table 1).

In the reliability test-retest, eligible community living older persons were recruited from the Gothenburg region. Older persons that had finalized geriatric rehabilitation or a continuum of care intervention were asked to participate. 22 In total, 39 of the 45 invited persons participated. The persons who declined to participate had either poor health or had no interest in participating (Table 2).

\section{Validity of the IPA-S \\ Procedure and analysis}

All questions from the IPA-S were read out loud in both the focus groups and in the individual interviews. Both focus groups and the individual interviews focused on whether each

item was comprehensible and relevant. Also, the participants were asked about their overall impression of the questionnaire. The sessions were recorded and transcribed verbatim. The focus group discussions were analysed based on the Krueger's method. ${ }^{23}$ Content analysis was used for analyzing the individual interviews. ${ }^{24}$ Relevant sections in the focus groups discussions were identified and sorted according to the aim of the study and the analysis was founded on how the participants discussed matters and the meaning that emerged from that discussion. For the individual interviews, the content analysis was performed with manifest content by looking for meaning units. Thereafter, meaning units from both the focus group discussions and the individual interviews were summarized and analysed into categories by the expert panel. The validity test including the interviews performed at the service centre was performed by the last author and the findings were analyzed and discussed by the expert panel. The IPA-S was then adjust-

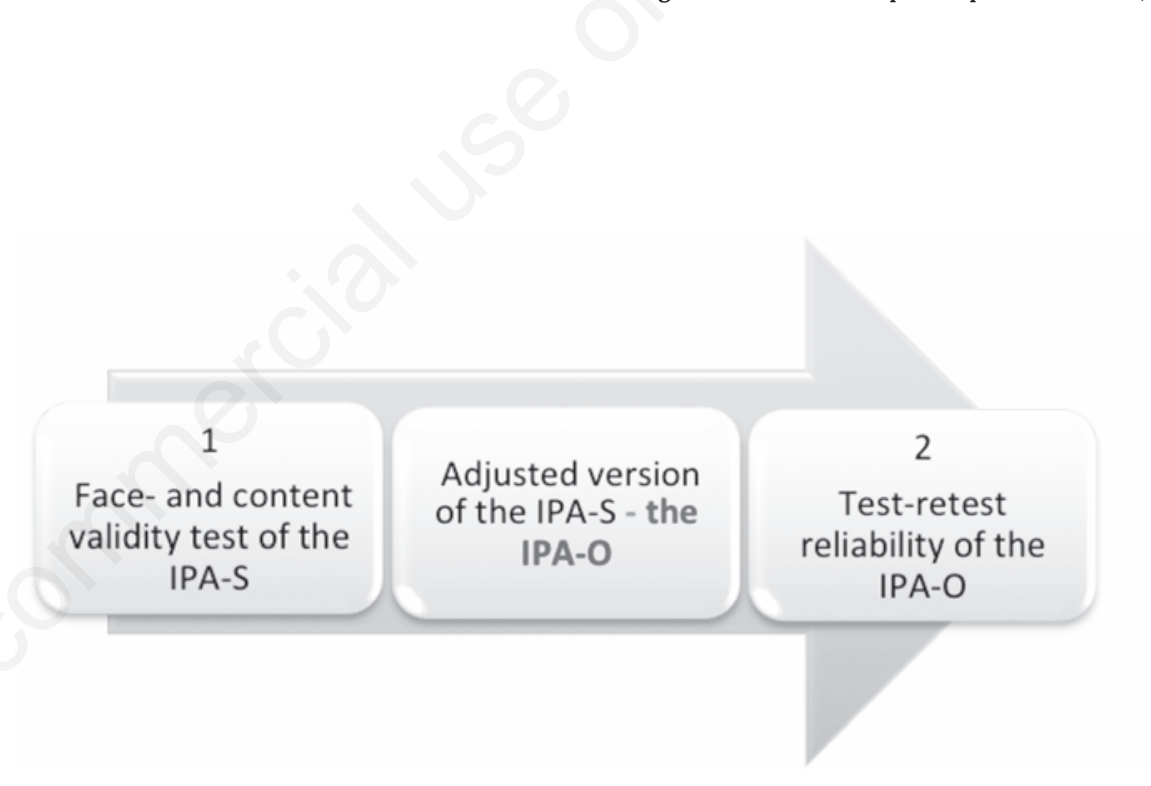

Figure 1. Research design.

Table 1. Characteristics of participants in the validity step.

\begin{tabular}{lcc} 
Participants $(\mathrm{n}=13)$ & $\mathrm{N}$. & $\%$ \\
Age, range (years) & $70-88$ & - \\
Gender, M/F & $3 / 10$ & $23 / 77$ \\
\hline Marital status, living alone & 13 & 100 \\
Housing, ordinary & 13 & 100 \\
\hline Dependence & & \\
$\quad$ I-ADL & 13 & 100 \\
I-ADL and P-ADL & 6 & 46 \\
\hline
\end{tabular}

I-ADL, Instrumental Activities of Daily Living; P-ADL, Personal Activities of Daily Living. ed by the expert panel resulting in IPA for Older persons (IPA-0).

\section{Reliability of the IPA-O \\ Procedure}

The participants were contacted by phone and received information about the study. After the participants verbally agreed to participate and the written information about the study was presented, the first interview was conducted as a face-to-face interview in the participants' home. At the first interview the participants had an enlarged copy of the answering categories in front of them and the questions were asked in given order. About fourteen days after the first test, the participants were contacted by telephone and the retest was performed. In total, 37 interviews were conducted by telephone and two by face-to-face interviews for the participants who had hearing impairment. Before the retest was performed, the interviewer ensured that nothing had changed in terms of participants' health,

\begin{tabular}{|c|c|c|c|}
\hline Activities of Daily Living. & $\begin{array}{l}\text { Dependence } \\
\text { I-ADL } \\
\text { I-ADL and P-ADL }\end{array}$ & $\begin{array}{l}39 \\
19\end{array}$ & $\begin{array}{c}100 \\
49\end{array}$ \\
\hline
\end{tabular}

Table 2. Characteristics of participants in the test-retest step.

\begin{tabular}{lcc} 
Participants $(\mathrm{n}=39)$ & $\mathrm{N}$. & $\%$ \\
Age, range (years) & $70-99$ & - \\
Gender, M/F & $13 / 26$ & $33 / 67$ \\
\hline Marital status & & \\
$\quad$ Married/cohabiting & 9 & 23 \\
$\quad$ Living alone & 30 & 77 \\
Housing & & \\
$\quad$ Ordinary housing & 35 & 90 \\
$\quad$ Nursing home & 4 & 10 \\
\hline Dependence & & \\
$\quad$ I-ADL & 39 & 100 \\
$\quad$ I-ADL and P-ADL & 19 & 49 \\
\hline I-ADL, Instrumental Activities of Daily Living; P-ADL, Personal \\
Activities of Daily Living.
\end{tabular}


which could have affected their self-determination. No participant was excluded due to this. Again, the participants had the enlarged copy of answering categories in front of them and the interviewer read the questions in a given order. The test and retest were therefore conducted in a similar way. The first, second and last author did the first test. The retests were done by the first and last author.

\section{Statistical analysis}

The data were analyzed in accordance with Svensson's method, ${ }^{19,20}$ which is a rank-based statistical method developed for treatment of paired assessments of ordinal data. The method made it possible to identify and separately assess occasional and systematic disagreement. ${ }^{19,20}$ The software for Svensson's method version 1.1.2 was used. The test-retest evaluations comprised of the following measures: i) the percentage agreement $(\mathrm{PA})$ is a basic value for categorical pairs of data between two assessments. ${ }^{25}$ PA of $\leq 59 \%$ was considered as low percentage agreement, PA of $60-69 \%$ as moderate and PA of $\geq 70 \%$ as high percentage agreement. ii) The relative rank position (RP) is a measurement of systematic shift in categorical levels between two assessments ranging between -1 and 1 . The higher value on $R P$, the higher systematic group change. RP close to zero indicates negligible change over time. $19,20 \mathrm{~A} 95 \% \mathrm{CI}$ on RP indicates an significant change on group level. 20 iii) The relative rank variance (RV) is a measure of the observed individual variability. ${ }^{20} \mathrm{RV}$ range from 0 to 1 . Higher values are a signal of presence of individual variability. ${ }^{20} \mathrm{RV} \geq 0.1$ was considered as a cut-off for the evidence of systematic disagreement regardless of the $\mathrm{CI}$ value. A 95\% CI on RV indicates an significant change on individual level. ${ }^{20}$

The values of RP and RV were calculated in all 22 items. If PA had high percentage agreement (PA of $\geq 70 \%$ ) the RP and RV were not considered relevant.

\section{Ethical considerations}

The study followed the ethical principles for medical research. ${ }^{26}$ In both the validity test and reliability test-retest, all participants were informed of their right to withdraw from the study at any time, that all data would be handled confidentially, and that no individuals could be identified. All participants gave both oral and written consent.

\section{Results}

\section{Validity of the IPA-S and adjust- ments}

The participants overall impression of the questionnaire were that the questions were relevant and of importance, but too extensive. Three categories emerged: Valued that their voice could be heard, The questionnaire was extensive and Focus on the execution. The participants Valued that their voice could be heard about their possibility of deciding for themselves. They gave numerous examples of friends and acquaintances that were questioned or even opposed in their self-determination either by relatives or by routines in home health care. The individual habits in daily life were particularly hampered when participants were assisted by others. Both focus groups discussed the point in having the questionnaire, but concluded that it could pinpoint this shortage of self-determination.

Participant 2: I think there are many that hear; don't do that or don't do that. I think many have others that decide for them. But I do what I want.

Participant 3: Yes, but you can still do it yourself

Participant 2: Yes, but if I'm disabled I can't. There are those who have others that decide for them, they don't have courage to decide for themselves.

Interviewer: So, do you think it's good to have such a question?

Participant 3: Yes, yes.

Participant 1: Yes, yes.

(Focus group)

The participants expressed The questionnaire was extensive, and recommended some items to be removed. The dimension Work and Education was considered irrelevant. They argued that the dimension about family role had too many items; My chances of contributing to looking after my home the way I want to, My chances of fulfilling my role at home as I would like to, could be removed. Also, there were too many items in the domain Social relations. The quality of my relationship with people who are close to me and My chances of having an intimate relationship were recommended to be omitted. The item about intimate relationship was discussed thoroughly, even if it did not have to imply a sexual relationship; the participants recommended its removal. Participants also emphasized one item that should not be omitted since it was found extremely valuable; $M y$ chances of helping or supporting people in any way.

Interviewer: If we should cut down on the amount of questions, which ones should we erase?

Participants': Those about education and work. But the question about helping others is important. You feel satisfied; you've done a good thing.

\section{(Individual interview)}

The participant's Focused on the execution in the items and not on the decisional part. Both in the focus group discussions and in the individual interviews the participants had to be reminded that it was not their ability to perform the different items they assessed, but their chances to have them done the way they wanted or when they wanted.

Interviewer: My chances of getting washed and dressed when I want to are?

Participant: No I can't. Pulling down my pants, it's very difficult. And then, in the bathroom, getting them up again, it's very difficult. They pull them up (home care service personnel). (Individual interview)

IPA-S was adjusted in accordance with the participants' recommendations and the expert panels' analysis resulting in 22 items (Table $3)$. The items were adjusted from questions to statements and the response options were adjusted to; totally agree, partly agree, neither agree nor disagree, disagree, and totally disagree. The items about perceived health problems were seen as important and relevant, both for the participants and the expert panel and therefore valid for the study group. No adjustments were made.

\section{Reliability of the IPA-O}

The range of PA between the test and retest assessments of all 22 items differed from 54 to $100 \%$ (Table 3 ). In three of the seven dimensions all items showed high agreement (PA $\geq 70 \%$ ).

Fifteen items indicated high agreement (PA $\geq 70 \%$ ). No evidence of systematic disagreement was found for these items. Six items showed moderate agreement PA range from 61 to $69 \%$, item $11-13,15,21-22$. The RP in item 11 indicated a change between the two tests at group level and the RV was close to zero demonstrating negligible presence of individual variability. The RP in item 12 indicated a decline of self-determination and the RV did not cover the zero value of the measures. In item 13, only 26 of the 39 participants answered. In item 11-13, both RP and PV had positive values with positive $95 \%$ CI values, indicating that the study group had scored higher perceived self-determination in the first test, than in the retest. In item 15, a systematic disagreement at group level was shown, towards a higher level of self-determination at the retest and the RV indicated presence of reduced self-determination on individual level. In item 21 both the RP ( 0.03$)$ and RV (0.04) were negligible small, indicating a reduction of self-determination on both group and individual level. In item 22, RP indicated a change at group level, with non-zero values of 95\% CI and the RV (0.03) demonstrated negligible presence of individual variability, with a reduced self-determination of the total perception after the retest.

One item showed low agreement with PA of $54 \%$ (item 4). The RP indicated an increase of self-determination and the RV was on the cut- 


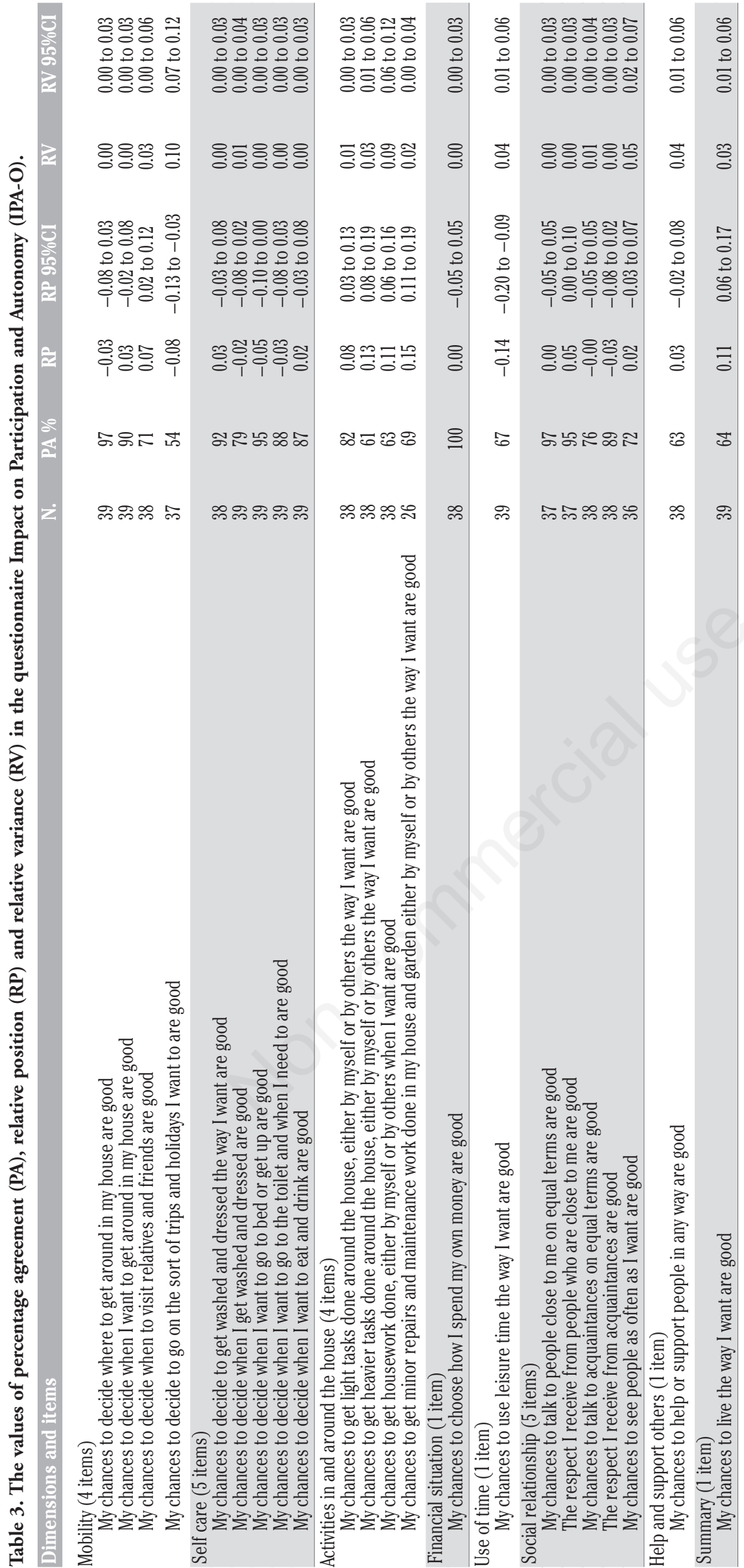

off limit ( $R V \geq 0.10$ ) with a $95 \%$ CI 0.07 to 0.12 indicating a significant presence of individual variability.

\section{Discussion}

In the validity part, the participants overall impression of the IPA-S questionnaire was that the questions were relevant and they emphasized being asked about their decision making in daily activities, but the questionnaire was too extensive. The participants' answers revealed that they focused on the executional and not on the decisional part of the activities in the questionnaire. Due to this, IPA-S was adjusted to a version called IPA-0, with fewer items with the decisional part of self-determination emphasized. The testretest results indicated that IPA-0 is a reliable questionnaire in 15 of the 22 items. Six items had moderate agreement and only one indicated low agreement. In item 11-13, dimension Activities in and around the house, both the RP and RV showed positive values which indicated that the participants' self-determination decreased between the two tests. The conclusion may be drawn that the participants after the first test had reconsidered their self-determination to be more reduced in the decision making process than before, in heavier tasks, housework or repairs. In the original version of IPA, items 11 and 13 had moderate agreement, ${ }^{14-16}$ which means that the present result is consistent with earlier.

Only the item 4, dimension Mobility, was considered to have low agreement (PA of $\leq 59 \%$ ). The values of RP indicated that the participants' self-determination had increased after the first test on group level and the RV indicated a reduction in perceived self-determination after the first test on individual level. The low agreement and the contradiction between the values can be explained by the complexity in some of the items in IPA-0, which deal with a wide area of daily activities. The complexity vary from less complex; $M y$ chances to decide when I want to go to bed or get up are good, to more complex; My chances to get housework done, either by myself or by others when I want are good. The items with moderate PA values are of the latter category and thus probably explain these lower values. More precise items could increase the PA values but would at the same time narrow down the area of relevance and possibly hamper the validity of the questionnaire. Consequently, the items with moderate PA values are recommended to be kept without change. The item with low PA; My chances to decide to go on the sort of trips and holidays I want to are good is even more complex item since it includes both trips and holidays, these activities can involve 
different ways of travelling each time. Still, even this item is kept based on the importance of the item as perceived by the target group but it is recommended to be interpreted with caution. There is a need for an instrument that assesses older persons' own perspectives and possibilities to be self-determined even when getting dependent on others, both clinically in rehabilitation and for research purposes. ${ }^{17}$ One limitation with the IPA-S, was that the participants answered the questionnaire emphasizing the executional part of daily activities and not both the decisional and executional part as intended by Cardol.13 Thus, it was imperative to rephrase the questionnaire but still observing the intent of IPA. The intent has been kept in spite of rephrasing from questions to statements. In addition, spontaneous feed-back by the participants in the testretest part of the study reflects that the decisional part is now more clearly noticeable.

The adjustments of the IPA-S resulted in fewer items based on the participants' comments and expert panels' analysis. There are several advantages by using fewer questions for this study population as expanded questionnaires can be exhausting to answer. There is a risk by decrease the amount of questions, but the older persons commented that some of the questions were not of relevance for them. Consequently, the benefits are greater than the risks.

The participants were few, which is a major limitation. Hence, the results must be interpreted with caution. Nevertheless, the characteristics of the participants indicate that they reflect the target group of older persons with affected ability to perform ADL independently. The participants' ability varied from having support several times a day to more occasional. All participants were assessed as being cognitively intact; no formal assessment was performed but the healthcare professionals performing the clinical assessment knew them well. Thus, further psychometric studies are needed to examine the usefulness of IPA- 0 for cognitively affected older persons. A strength with the validity test was that two data collection methods were used. In addition, a multiprofessional expert panel was used to analyse the focus groups' discussions and the individual interviews, as recommended in face and content validity tests. ${ }^{27}$ Thus, a variety of perspectives was obtained strengthening the triangulation.

The composition of the expert panel, could have influenced the design of IPA-0, but as their analysis is based on the participants' comments of the questionnaire and the expert panel are covering a broad relevant expertise area of frail older persons it is not considered as a major limitation. To test the reliability, a rank-invariant method by Svensson was used.19,20 Another method often used for ordi- nal data is Cohen's Kappa..$^{28}$ A comparison of the Kappa statistics and the rank-invariant method, showed that the rank-invariant method was superior when identifying and separately measuring systematic and random disagreement between test and retest. ${ }^{29}$ The time interval between the test and retest was 14 days, which is considered appropriate since the time is long enough for the persons to have forgotten the questions. ${ }^{30}$ Moreover, the time interval was appropriate in order to reduce the risk of further deterioration of the participants' health due to their frailty. The study group in the reliability test-retest consisted of older persons that were dependent on another person in at least one daily activity. Older persons with multiple diseases and decreased ability in daily activities are a complex group and their health situation can change radically. To ensure that this was not the case the participants were asked about whether their health had changed between the test and retest in as far as it could have affected their self-determination. No one was excluded on this account, but it still cannot be dismissed that their own perception of their self-determination can have been affected during this time interval.

\section{Conclusions}

The validity test showed that the questions in IPA-S were important, but some could be erased. To capture the decisional part of autonomy, the items were rephrased to make it clearer. IPA-S was revised into IPA-0.

The reliability test-retest of IPA-0 showed that the 15 items that had high agreement are reliable. The additional seven items are important aspects when assessing older persons' self-determination. Therefore, we recommend their inclusion but they must be interpreted with caution.

IPA-0 can be used for older dependent persons when planning their healthcare and rehabilitation to ensure their right to self-determination and participation. The questionnaire is recommended for use in all levels of rehabilitation, health care and in social care for the age group and is appropriate in the scientific research area when the purpose is to examine and investigate the older populations' perceptions of their self-determination.

\section{References}

1. United Nations. Charter of the United Nations. 2013. Available from: http://www.un.org/en/documents/charter/.

2. EUSTaCEA-project. European Charter of the Rights and Responsibilities of Older
People in Need of Longterm Care and Assistance. Available from: http://www.ageplatform.eu/images/stories/22204_AGE_ch arte_europeenne_EN_v4.pdf

3. Kristensson J, Hallberg IR, Ekwall AK. Frail older adult's experiences of receiving health care and social services. J Gerontol Nurs 2010;36:20-8.

4. Flick U, Fischer C, Neuber A, et al. Health in the context of growing old: social representations of health. J Health Psychol 2003;8:539-56.

5. Levasseur M, St-Cyr Tribble D, Desrosiers J. Meaning of quality of life for older adults: importance of human functioning components. Arch Gerontol Geriatr 2009;49:91-100.

6. Janlöv AC, Hallberg IR, Petersson K. Older persons' experience of being assessed for and receiving public home help: do they have any influence over it? Health Soc Care Community 2006;14:26-36.

7. Ekdahl A, Andersson L, Wirehn AB, Friedrichsen M. Are elderly people with comorbidities involved adequately in medical decision making when hospitalised? A cross-sectional survey. BMC Geriatr 2011;11:46-54.

8. Boyle G. Facilitating choice and control for older people in long-term care. Health Soc Care Community 2004;12:212-20.

9. Teeri S, Leino-Kilpi H, Valimaki M. Longterm nursing care of elderly people: identifying ethically problematic experiences among patients, relatives and nurses in Finland. Nursing Ethics 2006;13:116-29.

10. English V. Medical ethics today: the BMS s handbook of ethics and law. Oxford, Blackwell Publishing BMJ Books; 2004.

11. Elander G, Hermeren G. Autonomy and paternalistic behaviour in care. Scand J Caring Sci 1989;3:153-9.

12. Collopy BJ. Autonomy in long term care: some crucial distinctions. Gerontologist 1988;28:10-7.

13. Cardol M, De Jong BA, Ward CD. On autonomy and participation in rehabilitation. Disabil Rehabil 2002;24:970-4.

14. Cardol M, de Haan RJ, van den Bos GA, et al. The development of a handicap assessment questionnaire: the impact on participation and autonomy (IPA). Clin Rehabil 1999;13:411-9.

15. Cardol M, de Haan RJ, de Jong BA, et al. Psychometric properties of the impact on participation and autonomy questionnaire. Arch Phys Med Rehabil 2001;82:2106.

16. Cardol M, Beelen A, van den Bos GA, et al. Responsiveness of the Impact on Participation and Autonomy questionnaire. Arch Phys Med Rehabil 2002;83:1524-9.

17. Lund ML, Fisher AG, Lexell J, Bernspång B. 
Impact on participation and autonomy questionnaire: internal scale validity of the Swedish version for use in people with spinal cord injury. J Rehabil Med 2007;39:156-62.

18. Patton MQ. Enhancing the quality and credibility of qualitative analysis. Health Serv Res 1999;34:1189-208.

19. Svensson E. Ordinal invariant measures for individual and group changes in ordered categorical data. Stat Med 1998;17:2923-36.

20. Svensson E, Schillberg B, Kling AM, Nystrom B. Reliability of the balanced inventory for spinal disorders, a questionnaire for evaluation of outcomes in patients with various spinal disorders. J Spinal Disord Tech 2012;25:196-204.

21. Sonn U, Grimby G, Svanborg A. Activities of daily living studied longitudinally between 70 and 76 years of age. Disabil
Rehabil 1996;18:91-100.

22. Wilhelmson K, Duner A, Eklund K, et al. Continuum of care for frail elderly people: Design of a randomized controlled study of a multi-professional and multidimensional intervention targeting frail elderly people. BMC Geriatr 2011;11:24.

23. Krueger RA, Casey MA. Focus groups: a practical guide for applied research. Thousand Oaks: Sage Publications; 2000.

24. Graneheim UH, Lundman B. Qualitative content analysis in nursing research: concepts, procedures and measures to achieve trustworthiness. Nurs Educ Today 2004;24:105-12.

25. Svensson E. Guidelines to statistical evaluation of data from rating scales and questionnaires. J Rehabil Med 2001;33:47-8.

26. WMA. World Medical Association Declaration of Helsinki: ethical principles for medical research involving human sub- jects. JAMA 2013;310:2191-4.

27. Switzer GE, Wisniewski SR, Belle SH, et al. Selecting, developing, and evaluating research instruments. Soc Psychiatry Psychiatr Epidemiol 1999;34:399-409.

28. Altman DG. Practical statistics for medical research. London: Chapman and Hall; 1991.

29. Bunketorp L, Carlsson J, Kowalski J, Stener-Victorin E. Evaluating the reliability of multi-item scales: a non-parametric approach to the ordered categorical structure of data collected with the Swedish version of the Tampa scale for kinesiophobia and the self-efficacy Scale. J Rehabil Med 2005;37:330-4.

30. Nunnally JC, Bernstein IH. Psychometric theory. New York: McGraw-Hill; 1994. 